\title{
Lack of Association Between Age and Coronary Artery Calcification (CAC): a Cross-Sectional Study
}

\author{
Sheharyar Minhas, MD ${ }^{1,2}$, Zakaria Almuwaqqat, MD³, Maira Malik, MD ${ }^{4}$, Kathie Wu, MS ${ }^{5}$, and \\ Kevin Barretto, $M S^{5}$ \\ ${ }^{1}$ Rollins School of Public Health, Emory University, Atlanta, GA, USA; ${ }^{2}$ Department of Medicine, Nazareth Hospital, Philadelphia, PA, USA; \\ ${ }^{3}$ Department of Medicine Emory University Hospital, Atlanta, GA, USA; ${ }^{4}$ Combined Military Hospital Lahore Medical College, Lahore, Pakistan; \\ ${ }^{5}$ Philadelphia College of Osteopathic Medicine, Philadelphia, PA, USA.
}

J Gen Intern Med 34(7):1094-5

DOI: $10.1007 / \mathrm{s} 11606-019-04851-9$

(c) Society of General Internal Medicine 2019

\section{INTRODUCTION}

Coronary artery disease (CAD) has both modifiable risk factors such as obesity, diabetes, hyperlipidemia, hypertension, and non-modifiable ones including age, ethnicity, gender, and family history. These factors predispose a person to develop coronary artery calcification (CAC) which can be used to measure the extent of coronary heart disease. Stroke shares similar risk factors to CAC which has been associated with progression of atherosclerotic disease in the cerebrovascular system. The presence of CAC acts as an independent stroke predictor in the general population and CAC scores are superior to carotid artery intima-media thickness (IMT) for prediction of CHD and CVD events, including stroke and TIA. ${ }^{1}$ CAC score improves prediction, discrimination, and reclassification of CVD and CHD risk better than carotid ultrasound measures.

Various techniques can be used to detect calcifications including non-contrast cardiac computed tomography (CT). These results have been shown to correlate well with the presence of coronary atherosclerotic plaques and with the presence and extent of CAD on angiography. ${ }^{2,3}$ The Agatston score can then be calculated based on the amount of calcium found in the coronaries on CT. The calcified areas are assigned density scores and the total score of each individual can be calculated by summing the scores of every calcified focus through all of the coronary arteries. ${ }^{4}$ Scores of 1 to 10 represent minimal calcification, 11-100 represent mild coronary stenosis, 101 to 400 represent moderate non-obstructive coronary artery disease, and greater than 400 represent high likelihood of at least one significant coronary stenosis $(>50 \%){ }^{5}$ Stroke is a major cause of morbidity and mortality. Stroke has similar risk factors to CAD such as diabetes, hypertension, hyperlipidemia, obesity, and physical inactivity. Studies have found that coronary calcium is heavily influenced by ethnicity, in addition to race and gender. The purpose of this study was to examine the relationship between age and CAC scores and the relationship between stroke and CAC scores.

Published online February 12, 2019

\section{METHODS}

We obtained a sample of 3241 patients from Emory data warehouse between 2011 and 2016 with an Agatston score obtained through chest CT. Clinical and demographic characteristics were obtained. After removing patients who had minimal calcification (CAC score < 8), we log-transformed CAC score and age, and then used a simple linear regression model to assess for correlation. Using a similar regression model, we then assessed for correlation between CAC score, age, and stroke.

\section{RESULTS}

In the model which evaluates the independent effect of age on $\mathrm{CAC}$, we found that there was no statistically significant association $(P=0.84)$ (Table 1). These results were verified by plotting log (age) and $\log (\mathrm{CAC})$ on a scatter plot which demonstrated no significant correlation $(\mathrm{H} 0: \mathrm{B} 1=0)$ (Fig. 1). A second model which evaluates the correlation between age and history of stroke also demonstrated no statistically significant association $(P=0.43)$.

\section{DISCUSSION}

Although prior research has primarily focused on the relationship between CAC relationship and cardiovascular events such as stroke and CVD, there should be continued examination of the prevalence of, and risk

Table 1 Independent Effect of Age and Combined Effect of Age and History of Stroke on CAC Score

\begin{tabular}{llll}
\hline \hline & $\boldsymbol{\beta}$ & $\mathbf{9 5 \%}$ CI & Linear regression model \\
& $(\boldsymbol{P}$ value $)$ & & \\
\hline Age & -0.08809 & - & $\log (\mathrm{CAC})=\beta 0+\beta 1 \log ($ age $)$ \\
& $(0.84)$ & 0.94328, & \\
Age* stroke & -2.71800 & 0.76709 & \\
& $(0.43)$ & 9.51526, & $\log (\mathrm{CAC})=\beta 0+\beta 1 \log ($ age $)$ \\
& & 4.07926 & \\
\hline
\end{tabular}




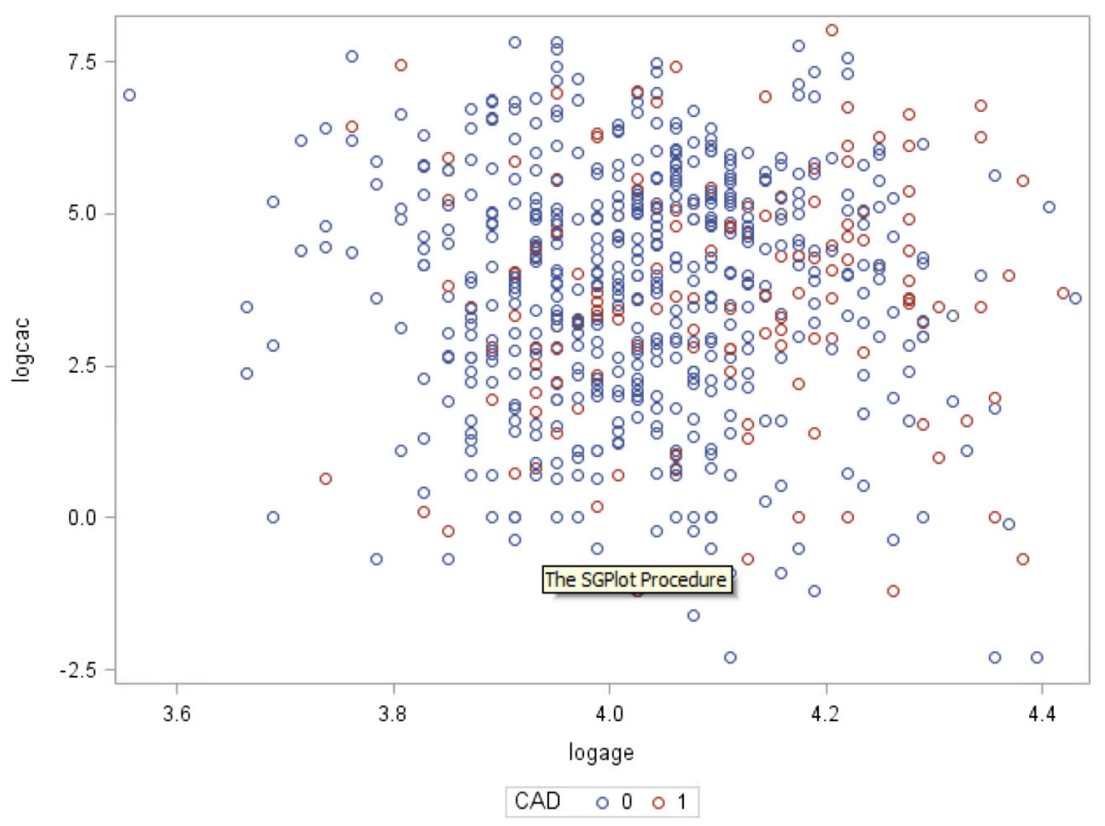

Figure 1 Scatter plot showing the correlation between $\log$ (age) and $\log$ (CAC score) stratified by CAD status

factors for, CAC. One study reiterates this point by discussing the lack of data on CAC evaluation in elderly patients and presented results that showed a strong association with age but a very wide range of calcification. $^{6}$ This outlines the importance of researching a possible age association with CAC, particularly in light of research that has linked CAC to CVD. Our data began as a large sample of patients with qualifying CAC scores which we analyzed based on age. Although our results show no statistically significant relationship between age and CAC or history of CVA, this presents an excellent basis for future examination, possibly by stratifying and accounting for other demographic factors. In this study, patients were only from the Emory data warehouse, predominantly Caucasian, and had to have presented with symptoms or medical history that warranted a chest CT. Our pool of non-CVA patients was also significantly smaller than those with a history of CVA. Further research is warranted with a larger sample size, more variation in demographic factors, and a greater range in age group. Continuing in this vein is critical and further quantitative analysis of CAC in relation to demographic data will help to illuminate its predictive value.

Corresponding Author: Sheharyar Minhas, MD; Department of Medicine Nazareth Hospital, 2601 Holme Avenue, Philadelphia, PA 19152, USA (e-mail: sminhas7@yahoo.com).

\section{Compliance with Ethical Standards:}

Conflict of Interest: The authors declare that they do not have a conflict of interest.

Publisher's Note: Springer Nature remains neutral with regard to jurisdictional claims in published maps and institutional affiliations.

\section{REFERENCES}

1. Gepner AD, Young R, Delaney JA, Tattersall MC, Blaha MJ, Post WS, Gottesman RF, Kronmal R, Budoff MJ, Burke GL, Folsom AR, Liu K, Kaufman J, Stein JH. Comparison of coronary artery calcium presence, carotid plaque presence, and carotid intima-media thickness for cardiovascular disease prediction in the Multi-Ethnic Study of Atherosclerosis. Circ Cardiovasc Imaging. 2015;8:e002262. https://doi.org/10.1161/ CIRCIMAGING.114.002262. Accessed 9 November 2018.

2. Rumberger JA, Schwartz RS, Simons DB, et al. Relation of coronary calcium determined by electron beam computed tomography and lumen narrowing determined by autopsy. Am J Cardiol. 1994;73:1169-1173. https://doi.org/10.1016/0002-9149(94)90176-7. Accessed 9 November 2018.

3. Otton JM, Yu CY, McCrohon J, et al. Accuracy and clinical outcomes of computed tomography coronary angiography in the presence of a high coronary calcium score. Heart Lung Circ. 2013;22:980-986. https://doi. org/10.1016/j.hlc.2013.05.647. Accessed 9 November 2018.

4. Agatston AS, Janowitz WR, Hildner FJ, Zusmer NR, Viamonte Jr M, Detrano R. Quantification of coronary artery calcium using ultrafast computed tomography. J Am Colle Cardiol. 1990;15(4):827-832. https:// doi.org/10.1016/0735-1097(90)90282-T. Accessed 9 November 2018.

5. Rumberger JA, Sheedy PF, Breen JF, Schwartz RS. Electron Beam Computed Tomographic Coronary Calcium Score Cut-Points and Severity of Associated Angiographic Lumen Stenosis. J Am Colle Cardiol. 1997;29(7):1542-1548. https://doi.org/10.1016/S0735-1097(97)000934. Accessed 9 November 2018.

6. Newman AB, Naydeck BL, Sutton-Tyrrell K, Feldman A, Edmundowicz D, Kuller LH. Coronary Artery Calcification in Older Adults to Age 99. Circulation. American Heart Association, Inc., 27 Nov. 2001. Web. 02 Apr. 2017. https://doi.org/10.1161/hc4601.099464. Accessed 9 November 2018 . 